\title{
«Commençons par connaître la réalité»
}

\section{Entretien: Bruno Kesseli}

Dr méd. et lic. phil., rédacteur en chef

Samia Hurst, médecin et bioéthicienne genevoise, participe au projet «Décisions médicales en fin de vie: fréquence et tendances en Suisse» dans le cadre du Programme national de recherche 67 «Fin de vie». A la Chambre médicale d'octobre dernier, elle a tenu un exposé très suivi à ce sujet. L'entretien qui suit revient sur certains aspects évoqués à cette occasion.

Que faut-il comprendre par "Décisions médicales en fin de vie» - pouvez-vous donner des exemples?

Dans le sens courant, les décisions de fin de vie incluent toutes les décisions qui se prennent en fin de vie qu'elles aient ou non un lien avec notre santé. Dans le sens utilisé dans les études sur les décisions de fin de vie, ce terme est plus précis. Il désigne toutes les décisions qui sont prises avec la conscience qu'elles pourraient raccourcir la vie, que ce soit ou non l'intention. Il désigne donc aussi toutes les décisions médicales prises dans le but de s'abstenir de prolonger la vie, ou dans le but de la raccourcir.

\section{Pourquoi est-ce important de disposer de données scientifiques sur les décisions en fin de vie?} La manière dont se déroule la fin de vie en Suisse nous informe sur plusieurs enjeux sociaux. Notre étude comparative suggère par exemple un recul de l'acharnement thérapeutique. Les situations de fin de vie font par ailleurs l'objet de controverses dans la médecine et la société. Il est important que ces débats se fondent sur des faits. Trop souvent, nous imaginons les situations, et ensuite nous jugeons si telle ou telle décision est bonne ou mauvaise. Notre imagination, cependant, est très limitée. Commençons par connaître la réalité.

\section{Dans quelle mesure le vif débat sur l'assistance} médicale au suicide a-t-il influencé le lancement de ce programme de recherche?

Il faudrait poser cette question aux personnes qui ont participé à la décision de lancer ce programme. Mon avis personnel est, cela dit, que l'influence aura été très limitée. Nous avons, c'est vrai, un débat durable en

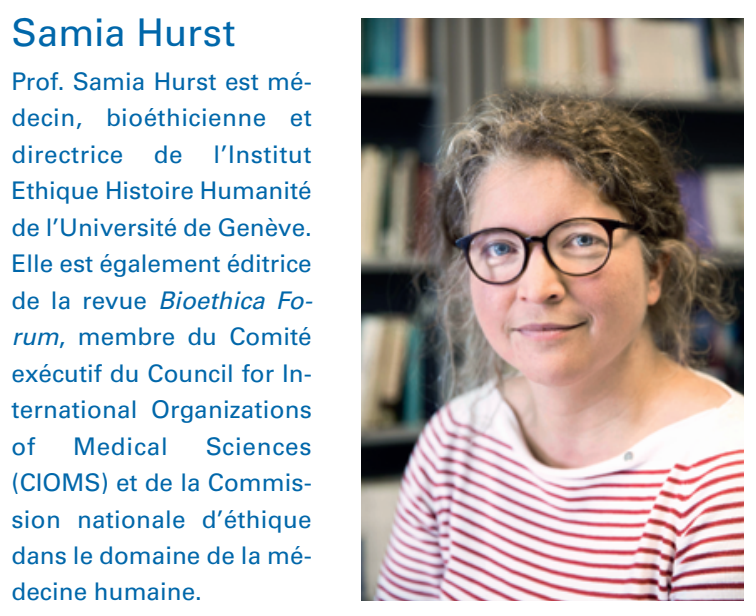

Suisse autour de la question de l'assistance au suicide. Le champ du PNR 67 est cependant nettement plus large. Il porte sur les conditions et sur les représentations de la mort en Suisse. C'est un sujet qui dépasse «Les situations de fin de vie font l'objet de controverses dans la médecine et la société. Il est important que ces débats se fondent sur des faits."

largement la question de l'assistance au suicide, qui ne concerne qu'une petite minorité des décès dans notre pays.

Pouvez-vous résumer brièvement les principales conclusions de l'étude?

Désormais, plus de la moitié des décès et $82 \%$ des décès attendus et non soudains sont précédés d'au moins 
une décision de fin de vie. La plupart de ces décisions concernent le retrait ou l'abstention thérapeutique, et l'intensification du traitement des symptômes. Cette évolution n'est pas homogène. L'abstention thérapeutique, la sédation palliative, et l'assistance au suicide ont augmenté. L'intensification du traitement des symptômes avec l'acceptation ou l'intention de hâter la mort n'a en revanche pas augmenté. Même si c'est elle qui fait l'objet des discussions les plus soutenues, l'assistance au suicide demeure très rare.

Y a-t-il des résultats qui vous ont surpris, vous et vos collègues? Si oui, lesquels?

Nous avons exploré l'implication des patients dans les décisions les concernant. La participation des patients capables de discernement a diminué.

«L'abstention thérapeutique, la sédation palliative, et l'assistance au suicide ont augmenté.»

\section{Comment expliquez-vous cette observation?}

Il est possible que l'on estime plus souvent qu'auparavant que poursuivre un traitement serait contraire à l'intérêt du patient. Dans ces situations, il est possible que des médecins estiment ne pas devoir proposer au patient des options qu'ils jugent contre-indiquées. Cette évaluation, cependant, devrait reposer sur les priorités du patient. Ce résultat semble donc problématique.

\section{Est-il possible de tirer des conséquences pratiques} directes à partir des données obtenues? Par exemple pour la formation prégraduée, postgraduée et continue des médecins ou pour leur activité pratique? Les décisions de fin de vie ont augmenté et doivent être mieux intégrées dans la formation pré- et surtout postgraduée des médecins. La place du patient dans ces décisions doit continuer d'être rappelée et précisée. Même si l'assistance au suicide reste rare, le fait qu'elle devienne plus fréquente va immanquablement soulever à nouveau la question de son intégration dans la formation médicale. Il ne s'agit pas de former les médecins à la pratique de l'assistance au suicide, car nul ne peut être tenu d'entrer en matière. En re-
PNR 67 «Fin de vie»

33 équipes de recherche étudient au sein du Programme national de recherche «Fin de vie» (PNR 67) des aspects liés à la fin de la vie à partir de diverses perspectives disciplinaires.

Le PNR a pour objectif de produire de manière scientifique des connaissances servant à définir des orientations ou des actions dans le domaine de la dernière phase de la vie et de les mettre à disposition des décideuses et décideurs du système de santé, de la politique et des professions qui s'occupent de la prise en charge des personnes en fin de vie.

Le PNR dispose d'une enveloppe de 15 millions de francs. Les recherches portent sur 5 ans.

Plus d'informations sur www.nfp67.ch/fr

vanche, un médecin doit être en mesure de prendre un certain nombre de précautions vis-à-vis d'un patient qui formule une demande d'assistance au suicide. Ces précautions pourraient, elles, faire l'objet d'une formation mieux ciblée.

\section{Comment les résultats seront-ils utilisés? Seront-ils par exemple repris en politique?}

L’Académie Suisse des Sciences Médicales revoit en ce moment sa directive médico-éthique sur la prise en charge des patients en fin de vie. Il est très utile que la plupart des données issues du PNR 67 soient disponibles pour le travail de cette sous-commission.

\section{Ce programme a-t-il soulevé de nouvelles questions qui seront éventuellement examinées lors de prochaines études?}

L'augmentation importante de la sédation palliative devra faire l'objet d'études plus approfondies, tant sur le plan factuel que sur le plan éthique.

«Les décisions de fin de vie ont augmenté et doivent être mieux intégrées dans la formation pré- et surtout postgraduée des médecins.»

\section{Quel est votre bilan personnel au terme du PNR "Décisions médicales en fin de vie»?}

C'est un bel exemple de ce qui est possible avec un financement public de la recherche autour d'un enjeu social important. 